\title{
Older recreational cross-country skiers adopt more even pacing strategies than their younger counterparts of similar performance level
}

\author{
Nikolaidis, Pantelis Theodoros ; Villiger, Elias ; Knechtle, Beat
}

\begin{abstract}
The aim of the present study was to examine the performance $\times$ age interaction on pacing in crosscountry (XC) skiing. We analyzed all finishers $(n=79,722)$ who competed in "Vasaloppet" from 2012 to 2017 grouped in performance quartiles according to their race time with Q1 as the fastest and Q4 as the slowest. Women $(44.1 \pm 10.2 \%)$ had larger pace range than men $(40.9 \pm 11.8 \% ; \mathrm{p}<0.001, \eta=0.014)$. The pace range ranged from $29.8 \pm 7.1 \%(\mathrm{Q} 1)$ to $49.0 \pm 10.1 \%(\mathrm{Q} 4 ; \mathrm{p}<0.001, \eta=0.179)$. In women and men, smaller differences in pace range among age groups for the faster performance groups were observed $(p<0.001, \eta=0.014, \eta=0.008$, respectively). In summary, fast XC skiers adopted relatively even pacing independent of their age, and the older $\mathrm{XC}$ skiers adopted more even pacing than their younger counterparts of similar performance level suggesting that differences among age groups are performance-dependent.
\end{abstract}

DOI: https://doi.org/10.1080/15438627.2018.1545647

Posted at the Zurich Open Repository and Archive, University of Zurich ZORA URL: https://doi.org/10.5167/uzh-165659

Journal Article

Accepted Version

Originally published at:

Nikolaidis, Pantelis Theodoros; Villiger, Elias; Knechtle, Beat (2019). Older recreational cross-country skiers adopt more even pacing strategies than their younger counterparts of similar performance level. Research in Sports Medicine, 27(3):365-373.

DOI: https://doi.org/10.1080/15438627.2018.1545647 
$1 \quad$ Older recreational cross-country skiers adopt more even pacing

\title{
strategies than their younger counterparts of similar performance
} level

\section{Running head: Pacing by age and performance in cross-country skiers}

\author{
Pantelis Theodoros Nikolaidis ${ }^{1}$, Elias Villiger ${ }^{2}$, Beat Knechtle ${ }^{2,3}$ \\ ${ }^{1}$ Exercise Physiology Laboratory, Nikaia, Greece \\ ${ }^{2}$ Institute of Primary Care, University of Zurich, Switzerland \\ ${ }^{3}$ Medbase St. Gallen Am Vadianplatz, St. Gallen, Switzerland
}

\section{2}

3

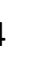

5

6

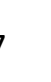

8

9

20

\section{Corresponding author}

Prof. Dr. med. Beat Knechtle

Medbase St. Gallen Am Vadianplatz

Vadianstrasse 26

9001 St. Gallen

Switzerland

Telefon +41(0) 712269300

Telefax +41(0) 712269301

E-Mail_beat.knechtle@hispeed.ch 


\section{$34 \quad$ Abstract}

The aim of the present study was to examine the performancexage interaction on pacing in cross-country (XC) skiing. We analyzed all finishers $(n=79,722)$ competing in 'Vasaloppet' from 2012 to 2017 grouped in performance quartiles according to their race time with Q1 the fastest and Q4 the slowest within each sex. A small main effect of sex on pace range was observed $\left(p<0.001, \eta^{2}=0.014\right)$, where women $(44.1 \pm 10.2 \%)$ had larger pace range than men $(40.9 \pm 11.8 \%)$. A large main effect of performance group on pace range was shown $\left(\mathrm{p}<0.001, \eta^{2}=0.179\right)$, where the smallest pace range was $29.8 \pm 7.1 \%(\mathrm{Q} 1)$ and the largest $49.0 \pm 10.1 \%(\mathrm{Q} 4)$. In women, a small age group $\times$ performance group interaction on pace range was shown $(\mathrm{p}<0.001$, $\eta^{2}=0.014$ ) with smaller differences in pace range among age groups for the faster performance groups. In men, a trivial age group $\times$ performance group interaction on pace range was shown $\left(\mathrm{p}<0.001, \eta^{2}=0.008\right)$ with smaller differences in pace range among age groups for the faster performance groups. In summary, fast XC skiers adopted a relatively even pacing independently from their age, and the older XC skiers adopted more even pacing strategies than their younger counterparts of similar performance level suggesting that differences among age groups are performancedependent.

Keywords: Endurance exercise, Gender, Race speed, Sport performance, Winter sport 


\section{Introduction}

An increased number of older athletes participate in endurance sports such as running, cycling, swimming and cross-country skiing (XC). XC skiing is popular in North and Central Europe, as well as in Canada, Russia and the United States of America (Nikolaidis, Heller, \& Knechtle, 2017).

Considering the impact of pacing on performance, developing an optimal pacing strategy is a major concern of endurance athletes (McCormick, Meijen, \& Marcora, 2016). The engagement of older XC skiers in regular training address the need for further research on this special group as limited information exists with regards to the effect of sex, age and performance on pacing (Carlsson, Assarsson, \& Carlsson, 2016; Formenti et al., 2015; Nikolaidis \& Knechtle, 2017c). Nevertheless, only three studies have examined the combined effect of sex, performance and age on pacing, and all three were conducted on the same sport (e.g. marathon running) and race (i.e. $\mathrm{New}$ York City Marathon’) (Breen, Norris, Healy, \& Anderson, 2017; Nikolaidis \& Knechtle, 2017a, 2017b).

A review of studies examining the effect of sex and performance on pacing in endurance running and cycling concluded that athletes of a higher performance level showed a more even pacing than their counterparts with a lower performance level, and women had less variable pacing than men (Thiel, Foster, Banzer, \& de Koning, 2012). In 10 and $15 \mathrm{~km}$ XC skiing races in World Cup, World Championships and Olympic events, slower male skiers were characterized by a relatively fast start, but no difference was found in women (Losnegard, Kjeldsen, \& Skattebo, 2016). A study 
on a relatively small sample of finishers in the 'Vasaloppet' skiing race showed that women had a more even profile than men (Carlsson et al., 2016).

As a result of this limited available information on the agexperformance interaction on pacing, coaches and fitness trainers working with older XC skiers currently rely on evidence resulting from other endurance sports in order to prescribe pacing strategies for training or competition. The knowledge of the variation of pacing by sex, age and performance in XC skiing would be of great practical relevance, especially considering the relationship of pacing with race time and perceived mental and physical strain during exercise (Venhorst et al., 2018). In addition, pacing has been considered essential to prevent premature fatigue prior to finishing an endurance race (Skorski \& Abbiss, 2017).

Thus, the aim of the present study was to examine the combined effects of age and performance on pacing in order to provide evidence-based suggestions for pacing in older XC skiers according to their performance level. We considered the example of the 'Vasaloppet' which is the largest XC skiing race in the world (Hållmarker et al., 2015). Based on the limited relevant research in XC skiing (Carlsson et al., 2016; Nikolaidis \& Knechtle, 2017c) and the extensive literature on endurance running, we hypothesized that older XC skiers would present a relatively even pacing compared to their younger counterparts independently from their performance level. 
104

105

106

107

108

109

110

111

112

\section{Methods}

To study the agexperformance interaction on pacing, the 'Vasaloppet', the oldest and longest cross-country ski race in the world with the largest rates of participation (Carlsson et al., 2016), was analyzed. The race had the full distance of $90 \mathrm{~km}$ with start in Sälen and finish in Mora, and included seven stations which defined eight splits (Figure 1). We obtained all data from the official race website (http://www.vasaloppet.se/) concerning 2012-2017, which were the calendar years with available full data about the age of finishers and split times. An initial screening of these data resulted in the final consideration of 79,722 finishers. It should be highlighted that data from races prior to 2012 were excluded from the present analysis as they did not report both age of finishers and split times. In addition, cases $(n=73)$ with at least one missing split time were excluded, too. All finishers were classified into age groups 19-20, 21-34, 35-39, 40-44, 45-49, 50-54, 55-59, 60-64, 65-69, 70-74, 75-79 and 80-84 year old. As there was sex difference in race time in XC skiing (Knechtle \& Nikolaidis, 2017; Nikolaidis \& Knechtle, 2017), finishers were classified into performance groups separately for each sex based on quartiles of race time $(\mathrm{Q} 1$, Q2, Q3 and Q4, with Q1 the fastest and Q4 the slowest). The dependent variable was the pacing strategy, whereas the independent variables were the sex, performance groups and age groups. To study pacing strategies, we calculated three pace parameters for each finisher (Breen et al., 2017): a) positive pace range in the fastest split as $100 \times$ (speed in the fastest split - mean race speed) / mean race speed, e.g. $+22.8 \%$, b) negative pace range in the slowest split as $100 \times($ speed in the slowest split - mean race speed) / mean race speed, e.g. $-16.5 \%$, and c) total pace range as the absolute difference between positive and negative range, e.g. $+22.8 \%-(-16.5 \%)=$ 
39.3\%. The institutional review board of "xxxxx" approved this study. Since the study involved analysis of publicly available data, the requirement for informed consent was waived.

All data were presented as means and standard deviations. Figures were created using GraphPad Prism v. 7.0 (GraphPad Software, San Diego, USA); all other statistical analyses were carried out using IBM SPSS v.23.0 (SPSS, Chicago, USA). Men-towomen ratio (MWR) was calculated for each age group as the number of men divided by the number of women. The sex $\times$ age group association and performance group $\times$ age group association was examined using chi-square $\left(\chi^{2}\right)$ and the magnitude of the associations was tested using Cramer's phi $(\varphi)$. The relationship of race speed with pace range, positive range and negative range was examined using the Pearson moment correlation coefficient $r$. Its magnitude was evaluated as trivial, $r<0.10$; small, $0.10 \leq r<0.30$; moderate, $0.30 \leq r<0.50$; large, $0.50 \leq r<0.70$; very large, $0.70 \leq r<0.90$; almost perfect $r \geq 0.90$ (Cohen, 1988). A two-way analysis of variance (ANOVA) examined the effects sex, performance group and age group on pace range. Subsequent comparisons among groups were carried out using post-hoc Bonferroni test. The magnitude of the differences among groups was examined using effect size eta square $\left(\eta^{2}\right)$ and was evaluated as following: small $\left(0.010<\eta^{2} \leq 0.059\right)$, moderate $\left(0.059<\eta^{2} \leq 0.138\right)$ and large $\left(\eta^{2}>0.138\right)($ Cohen, 1988). The acceptable type I error was set at $p<0.05$. 
154

155

156

157

158

159

160

161

162

163

164

165

166

167

168

169

170

171

172

173 174 in all cases for men.

175

176 A small main effect of sex on pace range was observed $\left(p<0.001, \eta^{2}=0.014\right)$, where 177 women $(44.1 \pm 10.2 \%)$ had larger pace range than men $(40.9 \pm 11.8 \%)$ (Figure 3). A 178 large main effect of performance group on pace range was shown $(p<0.001$,

\section{Results}

The total MWR was 7.10. A sex $\times$ age group association was observed $\left(\chi^{2}=1,297.7\right.$, $\mathrm{p}<0.001, \varphi=0.128$ ) with the MWR ranging from 3.38 (19-20 years group) to 54.13

(70-74 years group) (Table 1). In women, a performance group xage group association was shown $\left(\chi^{2}=159.2, \mathrm{p}<0.001, \varphi=0.127\right)$ (Figure 2). In men, a performance group $\times$ age group association was found $\left(\chi^{2}=2,246.1, p<0.001, \varphi=0.179\right)$.

In both sexes, we noticed that the prevalence of the quartile groups was more balanced in the younger age groups, whereas the slower groups (Q3 and Q4) were more prevalent in the older groups.

Pace range correlated largely with race speed in women $(r=-0.52, p<0.001)$ and men $(\mathrm{r}=-0.68, \mathrm{p}<0.001)$, i.e. the larger the pace range, the slower the speed. Positive range correlated moderately with race speed in women $(r=-0.41, \mathrm{p}<0.001)$ and largely in men $(\mathrm{r}=-0.55, \mathrm{p}<0.001)$, i.e. the larger the positive range, the slower the speed. Negative range correlated moderately with race speed in women $(r=0.48, p<0.001)$ and largely in men $(\mathrm{r}=0.63, \mathrm{p}<0.001)$, i.e. the larger the negative range (i.e. closer to zero), the faster the speed. In summary, all pace parameters (i.e. pace range, positive range and negative range) were related to race speed and this relationship was in the same direction in both sexes; however, the magnitude of this relationship was larger 
$\left.179 \eta^{2}=0.179\right)$, where the smallest pace range was $29.8 \pm 7.1 \%$ (Q1) and the largest

$18049.0 \pm 10.1 \%$ (Q4). A small sex×performance group interaction on pace range was

181 found ( $\left.\mathrm{p}<0.001, \eta^{2}=0.017\right)$ with men having the smallest pace range in Q1, Q2 and

$182 \mathrm{Q} 3$ and the largest in Q4.

183

184 In women, a small main effect of age group on pace range was observed $(\mathrm{p}<0.001$,

$\left.185 \eta^{2}=0.013\right)$ with age group 21-34 years showing the largest pace range $(44.8 \pm 10.9 \%)$

186 and age group 65-69 years the smallest (39.3 $\pm 8.6 \%$ ) (Figure 4A). A small age

187 group $\times$ performance group interaction on pace range was shown $\left(p<0.001, \eta^{2}=0.014\right)$

188 with smaller differences in pace range among age groups for the faster performance

189 groups. In men, a small main effect of age group on pace range was observed

$190\left(\mathrm{p}<0.001, \eta^{2}=0.026\right)$ with age group 19-20 years showing the largest pace range

$191(46.2 \pm 13.6 \%)$ and age group 70-74 years the smallest $(38.0 \pm 9.3 \%)$ (Figure 4B). A

192 trivial age group $\times$ performance group interaction on pace range was shown $(\mathrm{p}<0.001$,

$\left.193 \eta^{2}=0.008\right)$ with smaller differences in pace range among age groups for the faster

194 performance groups. Similar trends were observed in the other two parameters of

195 pacing (positive and negative range) that are shown in Figure 5.

196 
197

198

199

200

\section{Discussion}

The main findings of the present study were that $(i)$ fast older XC skiers had similar pacing as their younger counterparts, and (ii) older XC skiers had a more even pacing than their younger counterparts. Secondary findings were that $(i)$ the slower performance groups were more prevalent in the older age groups, (ii) the race speed correlated moderately to largely with all pacing indices, i.e. the faster the speed, the more even the pacing, and the magnitude of these correlations was larger in men, (iii) men had more even pacing than women in all performance groups except the slowest, (iv) age group 21-34 years showed the largest pace range and age group 65-69 years the smallest in women, whereas age group 19-20 years had the largest pace range and age group 70-74 years the smallest in men, and $(v)$ the MWR was larger in the older age groups.

The finding that older XC skiers presented a more even pacing than their younger counterparts was not surprising as it has been shown a similar trend in other endurance sports, except for $100 \mathrm{~km}$ running shwoing no differences in pacing among age groups (Rust, Rosemann, Zingg, \& Knechtle, 2015). For instance, a more even pacing has been observed in older runners in the 'New York City Marathon' (Nikolaidis \& Knechtle, 2017b). On the other hand, what was novel was that age did not influence the pacing strategies of the fast XC skiers indicating that fast older and younger XC skiers present similar performance characteristics (i.e. race time and pacing). It would be expected that since aerobic capacity, which is a main determinant of performance in XC skiing (Tonnessen, Haugen, Hem, Leirstein, \& Seiler, 2015), declines with aging (Rogers, Hagberg, Martin, Ehsani, \& Holloszy, 1990), older XC 
skiers would exhibit different performance characteristics (e.g. pacing) than their younger counterparts. Since fast XC skiers have a high aerobic capacity (Sandbakk, 2017) and are characterized by a relatively even pacing, it would be reasonable to assume that a relatively even pacing might be associated with a high aerobic capacity. Accordingly, a high aerobic capacity expressed as a high anaerobic threshold would assist XC skiers maintaining performance across race and preventing fatigue.

Nevertheless, training characteristics such as weekly training volume are predictors of the age-related changes in aerobic capacity (Kusy \& Zielinski, 2014; Rogers et al., 1990) indicating that training might attenuate the decrease of aerobic capacity. It should be highlighted that overall fast XC skiers presented a more even pacing in the present study, as it was shown by both the correlations between race time and pace range, and the comparison among performance groups. This finding was in agreement with findings in marathon runners (Breen et al., 2017; Nikolaidis \& Knechtle, 2017a,b). Nevertheless, the difference in pacing among performance groups decreased with age (Figure 4).

A surprising finding was that men had more even pacing than women, which was in contrast with previous findings on other endurance sports such as the 'Chicago Marathon' (Trubee, Vanderburgh, Diestelkamp, \& Jackson, 2014) and 100-km running (Renfree, Crivoi do Carmo, \& Martin, 2016) showing women as more even pacers. This discrepancy in the sex difference in pacing among endurance sports might be due to unique characteristics of XC skiing. Furthermore, the more even pacing in men observed in the present study was in disagreement with a previous study on a small sample of finishers in 'Vasaloppet', where women showed a more 
even pacing profile than men with the same finish time, start group, age, and race experience, and men were faster in the first half and women were faster in the second half of the race (Carlsson et al., 2016).

The results of the present study are limited by the unique characteristics of the 'Vasaloppet' in terms of race distance and change of elevation; therefore, they should be interpreted with caution when comparing with other $\mathrm{XC}$ races. Nonetheless, strength of the study was the inclusion of all editions of the 'Vasaloppet' (2012-2017) for which all split times and finishers' age were available resulting in one of the largest sample of XC skiers ever studied. The large number of finishers allowed drawing safe conclusions about differences in pacing by sex, age and performance group. Considering the large number of older XC skiers, the findings of the present study would be of practical importance for coaches and fitness trainers in this sport in order to adapt the training and competition practice such as pacing, which was previously established in younger XC skiers, in the specific demands of the older XC skiers. Professionals such as coaches, fitness trainers and physicians working with XC skiers provide services to athletes of a wide range of age and performance level of both sexes. Considering that adopting a pacing strategy is a major concern in this endurance sport (Carlsson et al., 2016; Karlsson et al., 2018), these professionals should provide evidence-based consultation depending on sex, age and performance level. Special attention should be drawn to master athletes, whose number of finishers in endurance races has increased during the last years relatively (\%) more than their younger counterparts (Lepers \& Stapley, 2016). 
271 In summary, based on the findings of the present study we identified a different effect

272 of age on pacing depending on the performance level of XC skiers. Fast older XC

273 skiers should be advised adopting a similar pacing strategy as their younger

274 counterparts. Older XC skiers should be expected to show a more even pacing than

275 their younger counterparts. Furthermore, we highlighted unique pacing patterns in XC

276 skiing which differ from other endurance sports such as the sex effect on pacing. Men

277 XC skiers have a more even pacing than women that is in contrast with the sex trends

278 in pacing in other endurance sports (e.g. running) that suggest women as more even

279 pacers. In XC skiing, the sex difference in pacing seems performance-dependent with

280 men showing more even pacing than women in all performance groups, except the

281 slowest.

283 Conflicts of interest

284 None declared.

286 Funding

287 No funding was received for this research. 
297

\section{References}

Breen, D., Norris, M., Healy, R., \& Anderson, R. (2017). Marathon pace control in masters athletes. Internation Journal of Sports Physiology and Performance, in print.

Carlsson, M., Assarsson, H., \& Carlsson, T. (2016). The influence of sex, age, and race experience on pacing profiles during the $90 \mathrm{~km}$ Vasaloppet ski race. Open Access Journal of Sports Medicine, 7, 11-19.

Cohen, J. (1988). Statistical power analysis for the behavioral sciences. Hillsdale, NJ: Lawrence Erlbaum Associates.

Formenti, D., Rossi, A., Calogiuri, G., Thomassen, T. O., Scurati, R., \& Weydahl, A. (2015). Exercise intensity and pacing strategy of cross-country skiers during a 10 km skating simulated race. Research in Sports Medicine, 23, 126-139.

Hållmarker, U., James, S., Michaëlsson, K., Ärnlöv, J., Sandin, F., \& Holmberg, L. (2015). Cancer incidence in participants in a long-distance ski race (Vasaloppet, Sweden) compared to the background population. European Journal of Cancer, 51, 558-568.

Hottenrott, K., Ludyga, S., Schulze, S., Gronwald, T., \& Jager, F. S. (2016). Does a run/walk strategy decrease cardiac stress during a marathon in non-elite runners? Journal of Science and Medicine in Sport, 19, 64-68.

Karlsson, Ø., Gilgien, M., Gløersen, Ø.N., Rud, B., \& Losnegard, T. (2018). Exercise intensity during cross-country skiing described by oxygen demands in flat and uphill terrain. Front iers in Physiology, 9, 846. 
Knechtle, B., \& Nikolaidis, P. T. (2017). The age of peak marathon performance in cross-country skiing - The 'Engadin Ski Marathon'. Journal of Strength and Conditioning Research, in print.

Kusy, K., \& Zielinski, J. (2014). Aerobic capacity in speed-power athletes aged 20-90 years vs endurance runners and untrained participants. Scandinavian Journal of Medicine and Science in Sports, 24, 68-79.

Lepers, R., \& Stapley, P.J. (2016). Master athletes are extending the limits of human endurance. Frontiers in Physiology, 7, 613.

Losnegard, T., Kjeldsen, K., \& Skattebo, Ø. (2016). An analysis of the pacing strategies adopted by elite cross-country skiers. Journal of Strength and Conditioning Research, 30, 3256-3260.

McCormick, A., Meijen, C., \& Marcora, S. (2016). Psychological demands experienced by recreational endurance athletes. International Journal of Sport and Exercise Psychology, in print.

Nikolaidis, P. T., Heller, J., \& Knechtle, B. (2017). The Russians are the fastest in marathon cross-country skiing: The "Engadin Ski Marathon". BioMed Research International, 2017, 9821757.

Nikolaidis, P. T., \& Knechtle, B. (2018). The age-related performance decline in marathon cross-country skiing - the Engadin Ski Marathon. Journal of Sports Sciences, 36, 599-604.

Nikolaidis, P. T., \& Knechtle, B. (2017a). Do fast older runners pace differently from fast younger runners in the 'new york city marathon'? Journal of Strength and Conditioning Research, in print.

Nikolaidis, P. T., \& Knechtle, B. (2017b). Effect of age and performance on pacing of marathon runners. Open Access Journal of Sports Medicine, 8, 171-180. 
Nikolaidis, P. T., \& Knechtle, B. (2017c). Pacing profiles in age group cross-country skiers in the vasaloppet 2012-2016. Chinese Journal of Physiology, 60, 293300 .

Renfree, A., Crivoi do Carmo, E., \& Martin, L. (2016). The influence of performance level, age and gender on pacing strategy during a 100-km ultramarathon. European Journal of Sport Science, 16, 409-415.

Rogers, M. A., Hagberg, J. M., Martin, W. H., 3rd, Ehsani, A. A., \& Holloszy, J. O. (1990). Decline in VO2max with aging in master athletes and sedentary men. Journal of Applied Physiology, 68, 2195-2199.

Rust, C. A., Rosemann, T., Zingg, M. A., \& Knechtle, B. (2015). Do non-elite older runners slow down more than younger runners in a $100 \mathrm{~km}$ ultra-marathon? BMC Sports Science, Medicine and Rehabilitation, 7, 1.

Sandbakk Ø. (2017). The evolution of champion cross-country-skier training: From lumberjacks to professional athletes. International Journal of Sports Physiology and Performance, 12, 254-259.

Skorski, S., \& Abbiss, C.R. (2017).The manipulation of pace within endurance sport. Frontiers in Physiology, 8, 102.

Thiel, C., Foster, C., Banzer, W., \& de Koning, J. (2012). Pacing in Olympic track races: Competitive tactics versus best performance strategy. Journal of Sports Sciences, 30, 1107-1115.

Tonnessen, E., Haugen, T. A., Hem, E., Leirstein, S., \& Seiler, S. (2015). Maximal aerobic capacity in the winter-Olympics endurance disciplines: Olympicmedal benchmarks for the time period 1990-2013. International Journal of Sports Physiology and Performance, 10, 835-839. 
368 Trubee, N. W., Vanderburgh, P. M., Diestelkamp, W. S., \& Jackson, K. J. (2014). Effects of heat stress and sex on pacing in marathon runners. Journal of Strength and Conditioning Research, 28, 1673-1678.

371 Venhorst, A., Micklewright, D., \& Noakes, T.D. (2018). Towards a three-dimensional framework of centrally regulated and goal-directed exercise behaviour: a narrative review. British Journal of Sports Medicine, 52, 957-966. 
374

375

\begin{tabular}{ccccc}
\hline Age group & Women & Men & Total & MWR \\
\hline $\mathbf{1 9 - 2 0}$ & 177 & 599 & 776 & 3.38 \\
$\mathbf{2 1 - 3 4}$ & 3,905 & 17,726 & 21,631 & 4.54 \\
$\mathbf{4 0 - 3 9}$ & 1,150 & 9,208 & 10,358 & 8.01 \\
$\mathbf{4 5 - 4 9}$ & 1,520 & 11,837 & 13,357 & 7.79 \\
$\mathbf{5 0 - 5 4}$ & 1,355 & 10,827 & 12,182 & 7.99 \\
$\mathbf{5 5 - 5 9}$ & 1,024 & 8,334 & 9,358 & 8.14 \\
$\mathbf{6 0 - 6 4}$ & 440 & 5,102 & 5,542 & 11.6 \\
$\mathbf{6 5 - 6 9}$ & 185 & 3,279 & 3,464 & 17.72 \\
$\mathbf{7 0 - 7 4}$ & 75 & 1,862 & 1,937 & 24.83 \\
$\mathbf{7 5 - 7 9}$ & 16 & 866 & 882 & 54.13 \\
$\mathbf{8 0 - 8 4}$ & 0 & 194 & 194 & \\
\hline $\mathbf{T o t a l}$ & $\mathbf{9 , 8 4 7}$ & $\mathbf{6 9 , 8 7 5}$ & $\mathbf{7 9 , 7 2 2}$ & \\
\hline & 0 & 41 & 41 & \\
\hline
\end{tabular}

Table 1 Finishers by sex and age group
376

377

378

379

380

381

382

383

MWR=men-to-women ratio 
$384 \quad$ Legends of figures

385

386 Figure 1 Race speed (\%) and change of elevation by split

387

388

Figure 2

Distribution of performance groups by age group

389

$390 \quad$ Figure 3

Pace (A), positive (B) and negative range (C) by sex and

391

performance group

392

$393 \quad$ Figure 4

Pace range by performance group and by age group in women

394

(A) and men (B)

395

$396 \quad$ Figure 5

Positive (A, B) and negative range (C, D) (\%) of speed by

397

performance group and by age group in women and men

398

399 


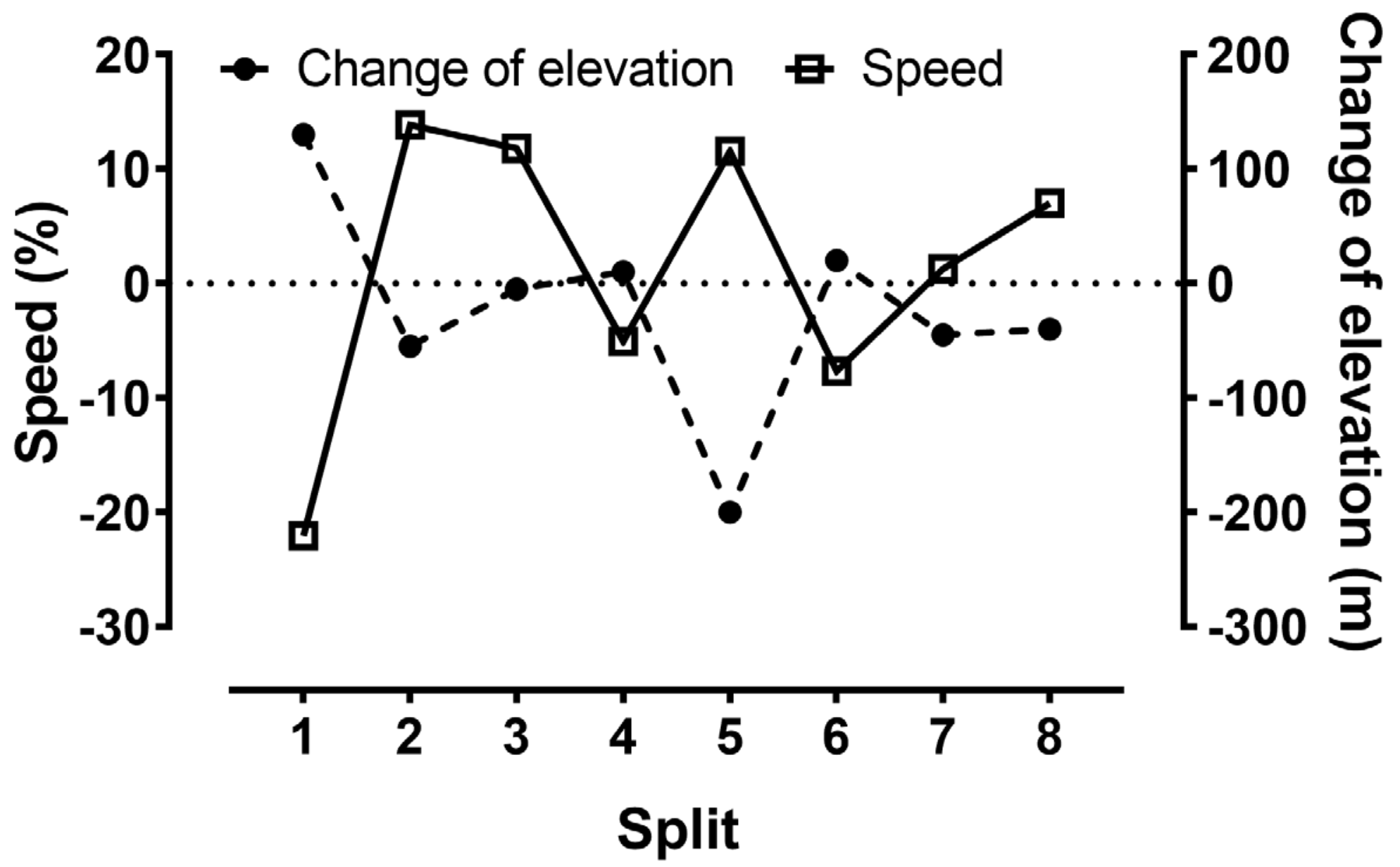



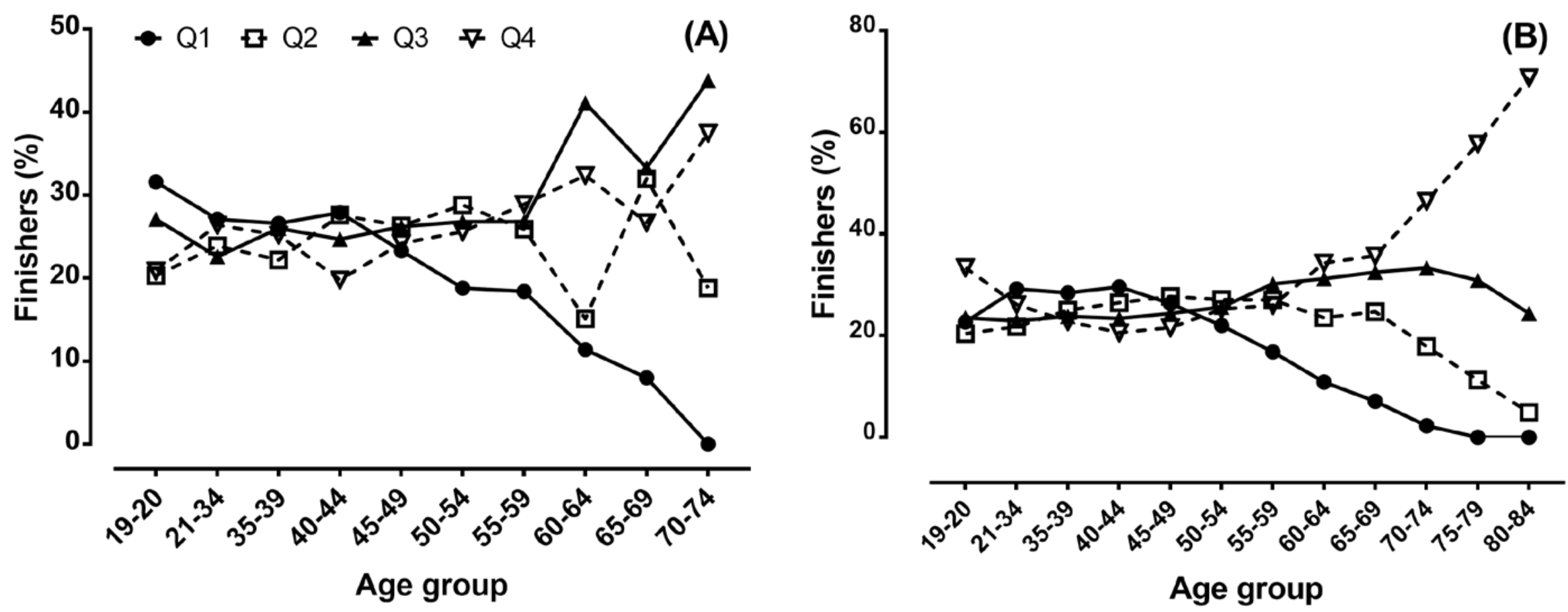
Figure 3
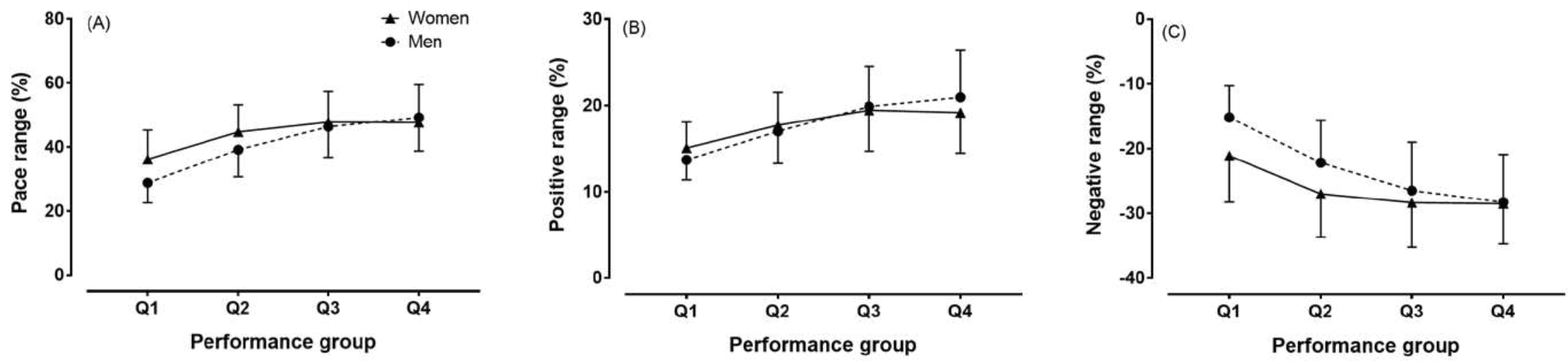
Women

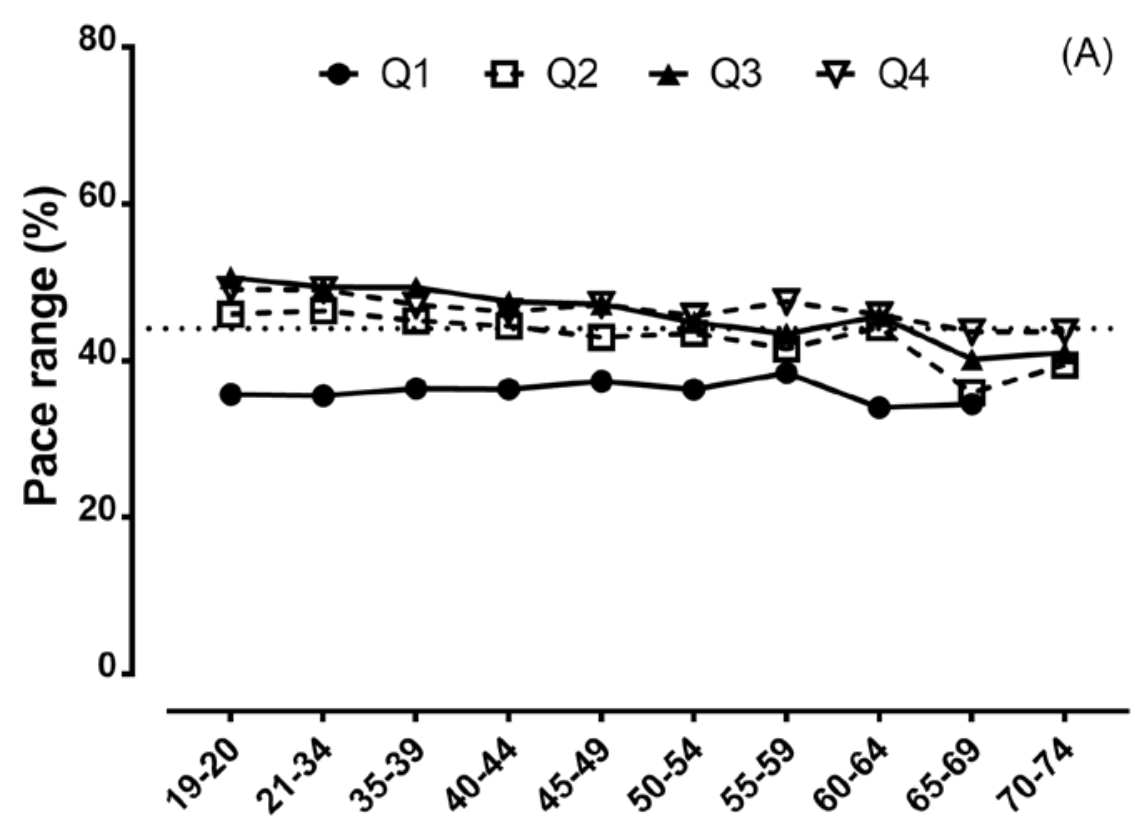

Age group

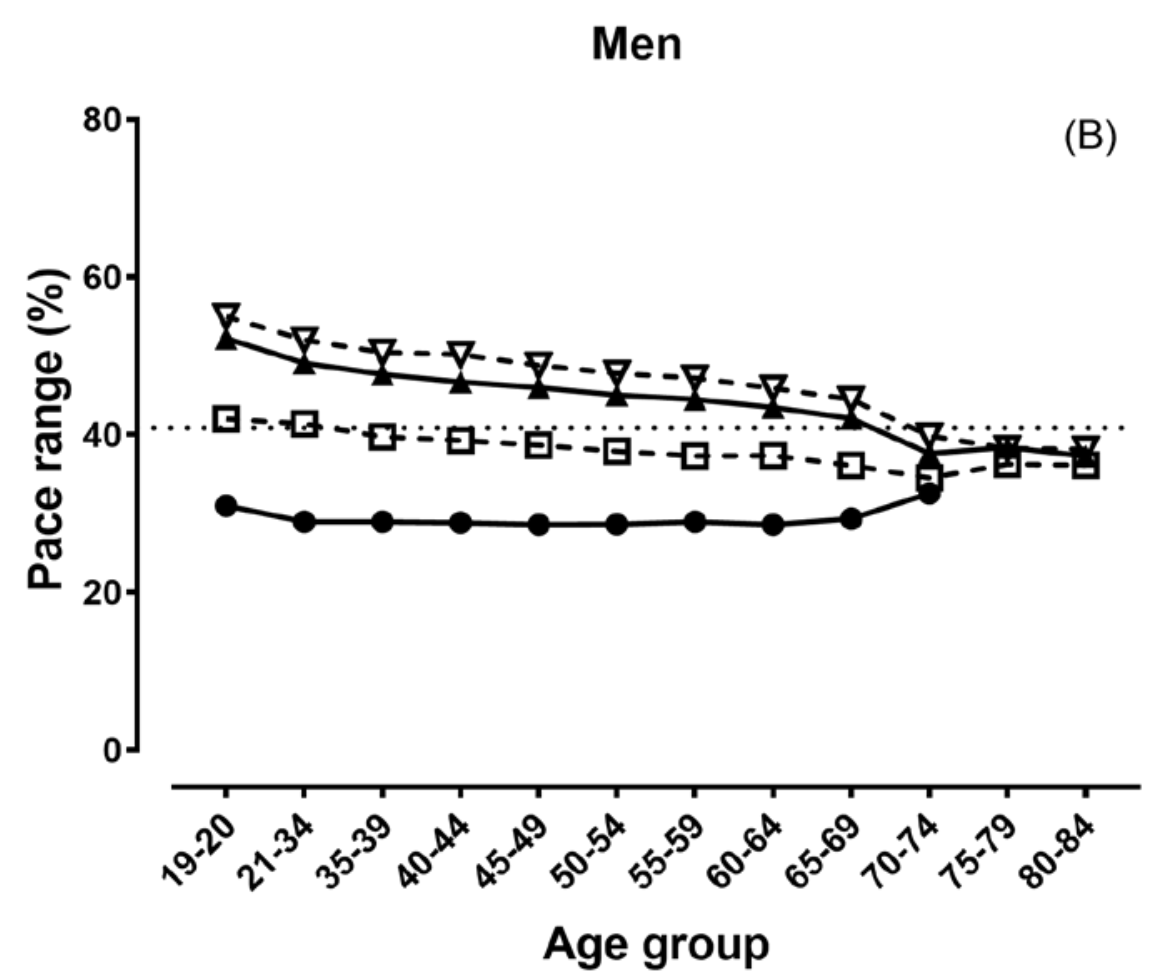

(B) 

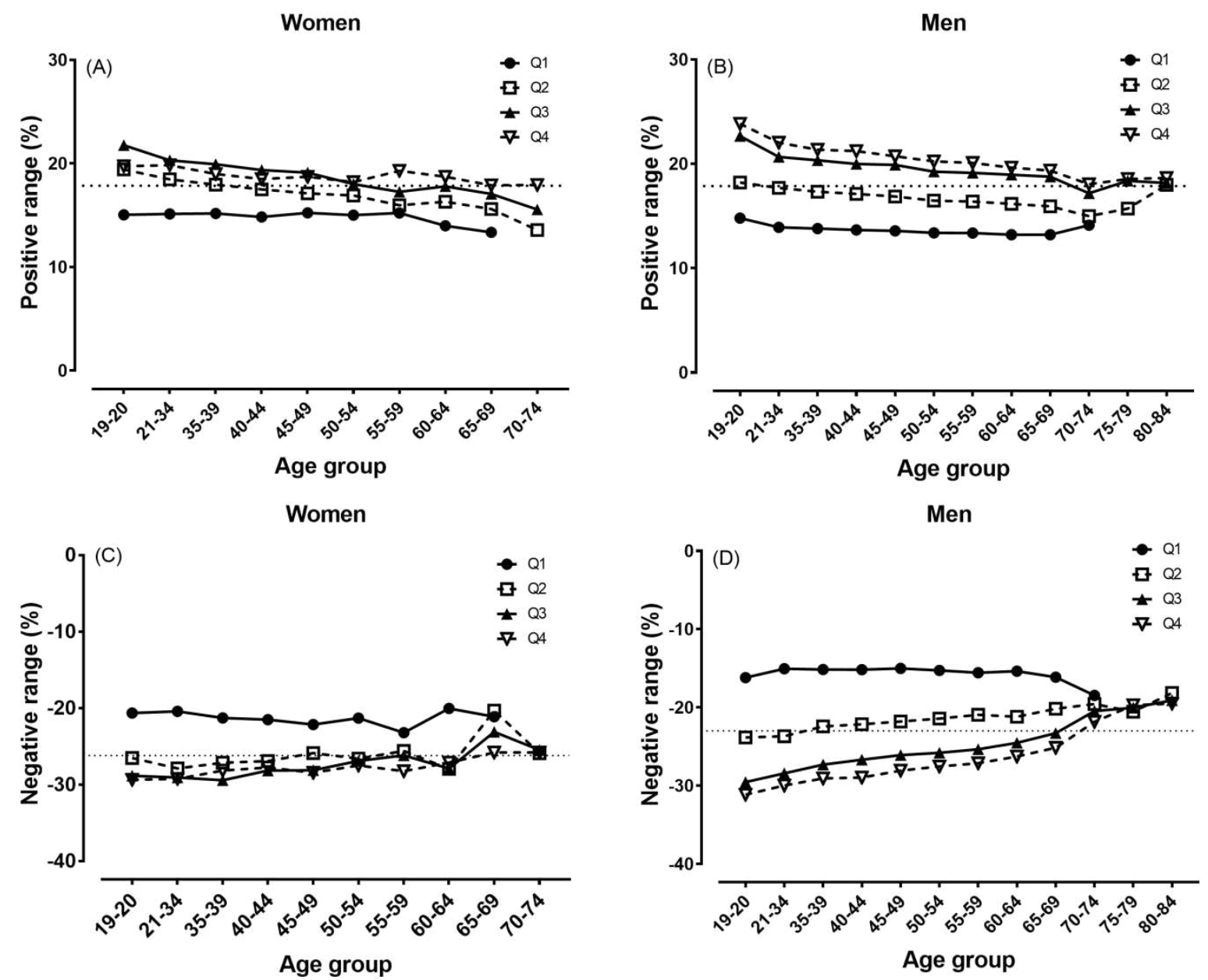